\title{
Friction and wear behaviour of Scapharca subcrenata under sliding contact conditions as a function of sliding speed and loading level
}

\author{
You. Lv ${ }^{1, \text { a }}$, Xiu.Fang. Zhang ${ }^{2, b}$, Wen.Cui. Xiu ${ }^{3, c}$, Li.Qun.Lei ${ }^{4, d}$, Li.Na.Sun ${ }^{5, e}$ \\ 1,2,3,4,5 School of Mechanical Engineering, Jilin Agriculture Science and Technology College, Jilin \\ City, Jilin Province, 132101, P.R. China \\ aemail:Ilvvyou@163.com, bemail:zhangxiufang@163,com, cemail:xiuwencui@163.com, email:leiliq \\ un@163.com, eemail:sunlina@163.com
}

Keywords: Friction; Wear; Biomaterials; Microstructure; Composite

\begin{abstract}
This paper investigates the microstructure and friction-wear behaviour of Scapharca subcrenata in order to obtain useful information regarding the tribological properties of composite materials. The experimental results have showed that the relationship between the friction rate and surface roughness of the friction pair was the most important. The wear rate of the friction pair decreased with a decrease in the surface roughness. The load level was the most influential factor to the friction coefficient of the friction pair, and the influence of test time on the friction coefficient was not significant. Scapharca subcrenata was dominated by abrasive wear mechanisms, and the wear loss of Scapharca subcrenata increased linearly with an increment of the loading level.
\end{abstract}

\section{Introduction}

Shell is a typical bioceramic composite material which is composed of 95\% 99\% of crystalline inorganic minerals (calcite or aragonite) and 1\% 5\% of organic collagen [1,2]. The microstructure of shell is significantly different due to different species and living environments. Even the same shell has different microstructure characteristics in different positions. A shell is usually composed of a cuticle, a prismatic layer and a pearl layer along the depth direction. The cuticle is the outermost layer of the shell, and it is mainly composed of hard protein. The prismatic layer is the middle layer of the shell, and it is mainly composed of calcite crystals. The pearl layer forms the inner layer of the shell, and it is mainly composed of aragonite crystals [3].

Through the investigation of the tribological behaviour and microstructure of the shell, it is helpful to enrich the bionic theory and to develop high-performance wear-resistant bionic materials $[4,5]$. Scapharca subcrenata has complex layered structures. The pearl and prismatic layers feature excellent mechanical properties and corrosion resistance. The research investigating the friction and wear behaviour of Scapharca subcrenata has played an important role to improve the tribological properties of layered composite materials. In this paper, different mesh sandpapers were used as friction pairs to study the tribological behaviour of Scapharca subcrenata under different load conditions. The worn surface was observed by a scanning electron microscope (SEM), and then the wear mechanism of Scapharca subcrenata was analysed.

\section{Experimental procedures}

Firstly, Scapharca subcrenata shells were cleaned by water, and then soaked in $5 \% \mathrm{NaOH}$ solution for $10 \mathrm{~min}$. Afterwards, the samples were cut into a size of $10 \mathrm{~mm} \times 15 \mathrm{~mm}$ by a precision cutting machine. The wear test was carried out on a friction and wear testing machine, as is shown in Fig.1. The loading levels were $15 \mathrm{~N}, 20 \mathrm{~N}$ and $25 \mathrm{~N}$, respectively. The sliding speeds were $120 \mathrm{r}$ $/ \mathrm{min}, 150 \mathrm{r} / \mathrm{min}$ and $180 \mathrm{r} / \mathrm{min}$, respectively. Meshes of friction pairs were 100\#, 240\# and 1000 \#, respectively. The test time was 300s, 600s and 900s respectively. Each test was repeated for 5 times, and the average value was the final result. 


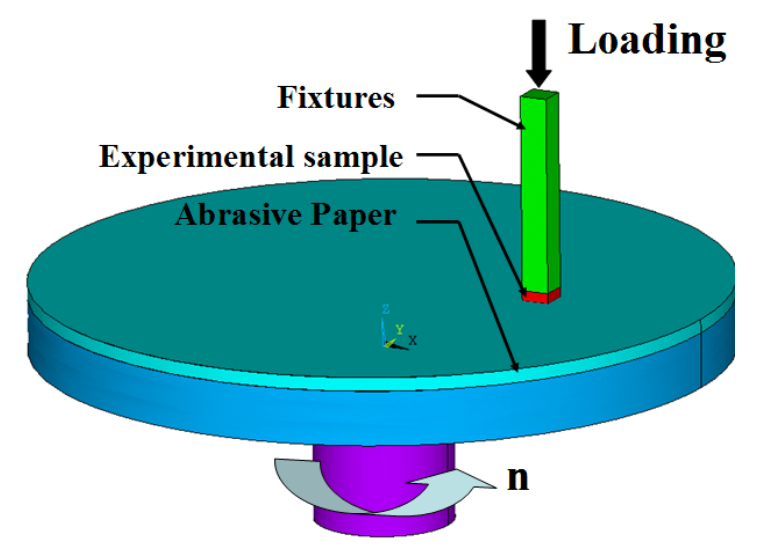

Fig.1. Schematic diagram of wear testing machine

\section{Results and discussions}

Surface morphology

Fig.2 shows the surface morphology of Scapharca subcrenata. Scapharca subcrenata shells have stripe ribs. The surface of the radial rib is smooth, and the rib is generally $500 \mu \mathrm{m}-700 \mu \mathrm{m}$ in height and $800 \mu \mathrm{m}-1200 \mu \mathrm{m}$ in width. The distance between the radiating ribs is generally $500 \mu \mathrm{m}-800 \mu \mathrm{m}$. The shell surface is white and has brown villous hairs.

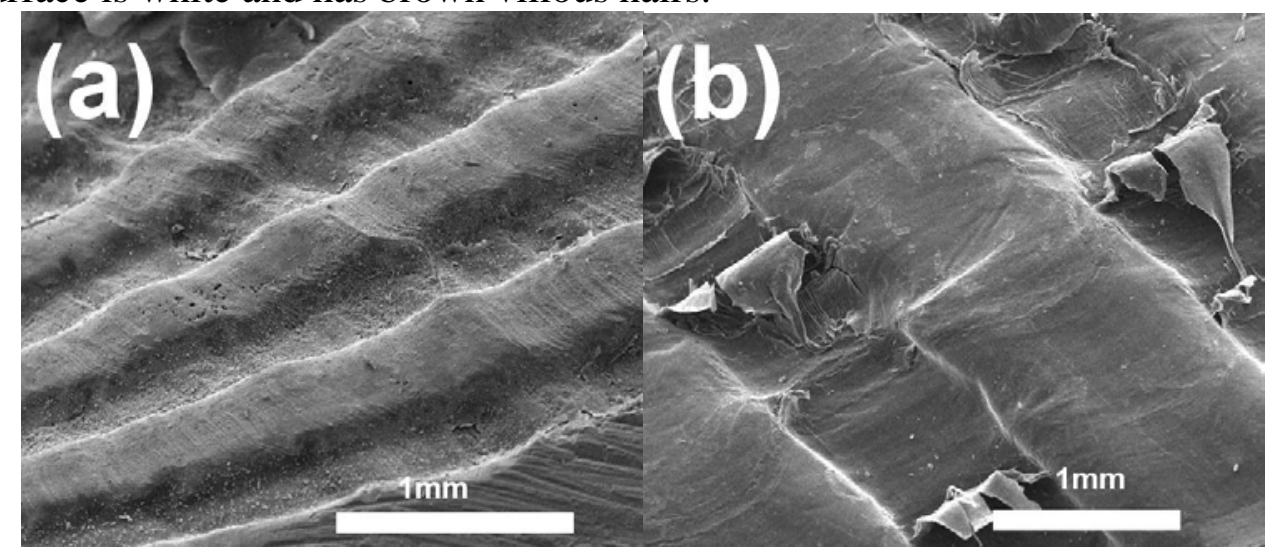

Fig.2. Scapharca subcrenata shell surface radial ribs and villous hairs

Microstructure

The longitudinal microstructure of Scapharca subcrenata from the outside to the inside is composed of multilayer structures, including the horny layer, prismatic layer, pearl layer and rubble layer. As shown in Fig.3, the prismatic layer occupies most of the Scapharca subcrenata shell. The whole prismatic layer is composed of columnar structures, and the adjacent columnar structures are vertically intersecting, presenting a typical cross-laminated structure. This kind of multi-oriented lamellar structure has greater strength than a single-orientation structure. When the multi-oriented lamellar structure is impacted by an external force, it could absorb large amounts of energy, effectively inhibiting micro crack propagation and fractures and thus improving the wear resistance and fatigue performance of Scapharca subcrenata. The columnar structure consists of many parallel lamella structures. The angle between the lamella and the direction of the columnar axis is about 60 degrees. Each lamella is formed by some crystals, of which the orientation is roughly parallel on the same lamella. 


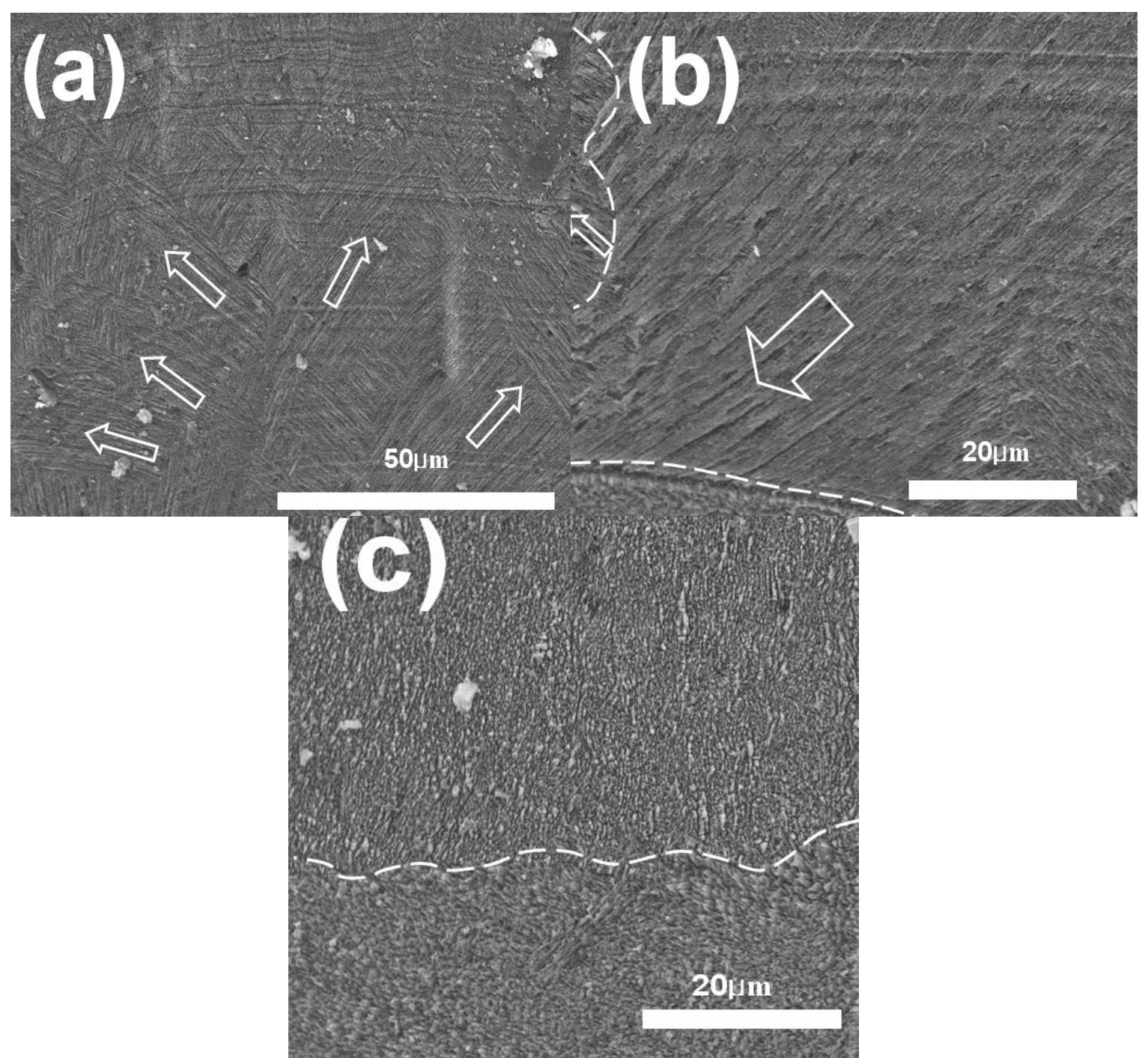

Fig.3. Microstructure of Scapharca subcrenata prismatic layer

The EDS result of Scapharca subcrenata powder is shown in Fig.4. It can be seen that the main elements are calcium, carbon and oxygen. From the XRD results in Fig.5, we can know that the main chemical composition of Scapharca subcrenata is calcium carbonate $\left(\mathrm{CaCO}_{3}\right)$. It is indicated that the phase composition of shell was aragonite mainly. Aragonite was widely existed in crystal structure, and played an important role in various functional properties of shell.

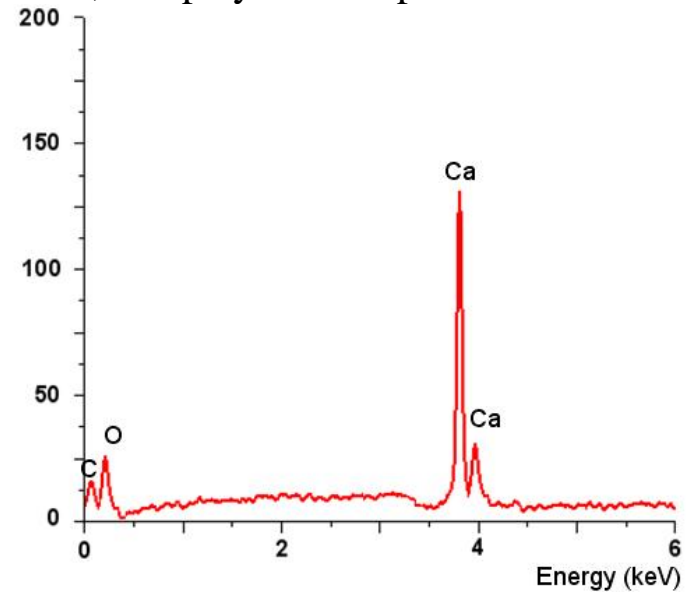

Fig.4. EDS analysis result of Scapharca subcrenata

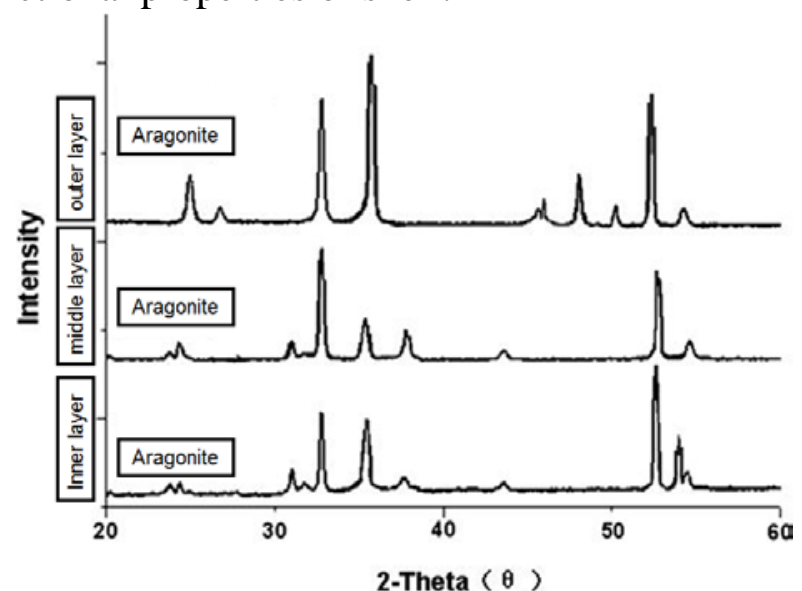

Fig.5. XRD analysis result of Scapharca subcrenata

Experimental results

Table 1 exhibits the orthogonal test results of the effect of speed, normal load and time on the friction coefficient and wear rate of Scapharca subcrenata. After performing a range analysis, it can 
be seen that the influences of each factor on frictional coefficients are the load $>$ speed $>$ friction time. At a certain speed, the frictional coefficients increased linearly with an increase in loads. The frictional coefficients were obviously different when the load changed. When the speed was $120 \mathrm{r} / \mathrm{min}$ or $180 \mathrm{r} / \mathrm{min}$, the frictional coefficients increased as the load increased. When the speed was $150 \mathrm{r} / \mathrm{min}$, the frictional coefficient firstly became larger and then decreased with an increase in load. Fig.6 shows the wear morphology of Scapharca subcrenata. There were two main wear mechanisms of shell materials: abrasive and adhesive wear. Visibly, Scapharca subcrenata was dominated by abrasive wear mechanisms, and the traces of the plough were obvious.

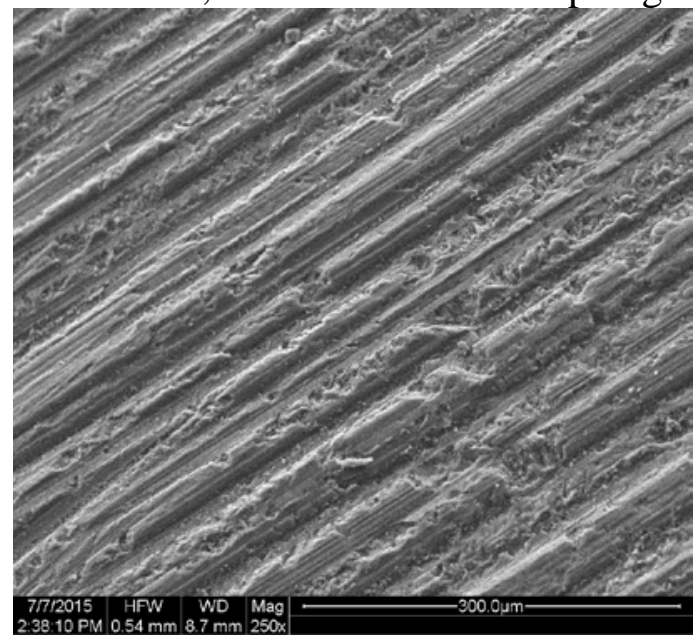

Fig.6. The wear morphology of Scapharca subcrenata

\section{Discussions}

Effect of microstructure on the frictional coefficient

According to Fig.3, the inorganic structure of Scapharca subcrenata is mainly composed of prismatic and nacreous layers, and the friction process was divided into 2 steps. Firstly nacreous aragonite contacted and rubbed with sandpaper. As the tests went on, the nacreous layer was exhausted, and then the prismatic layer contacted with sandpaper. By analysing the frictional coefficient, it can be indicated that a sudden decrease in the friction factor was because that the friction coefficient of the prismatic layer was less than that of the nacreous layer under the same test conditions. The compositions of the prismatic layer and nacreous layer were basically the same, i.e. $5 \%$ of the organic matter and $95 \% \mathrm{CaCO}_{3}$. The difference lies in that the nacreous layer consisted of aragonite, and the prismatic layer consisted of columnar calcite.

\subsubsection{Effect of experimental conditions on the frictional coefficient}

Under a certain load, the frictional coefficient decreased rapidly with an increase in the sliding speed, and finally tended to be stable. When the sliding speed was small, the engagement degree between the sandpaper and the radiation rib of Scapharca subcrenata was relatively large, and the frictional force and frictional coefficient were large. With an increase in sliding speed, the temperature of the Scapharca subcrenata surface increased while the hardness of aragonite platelets dropped, which caused plastic flow and planarisation of the nacreous layer. As the degree of engagement between the radial rib and sandpaper became smaller and smaller, the hindrance of mutual movement gradually weakened, which led to a decrease in the micro-cutting resistance of Scapharca subcrenata. Under the same loading conditions, the macro-friction resistance obviously decreased, and the frictional coefficient was reduced. Therefore, the frictional force and frictional coefficient of Scapharca subcrenata were closely related to the degree of engagement between radial rib and sandpaper.

Before 200s, the friction coefficient of Scapharca subcrenata was relatively large since the cuticle hardness was relatively high, and its rough surface led to an increase in the frictional force. After 200s, the experimental process entered the pure material wear stage, while the cuticle of Scapharca subcrenata was completely worn out. The bearing part of Scapharca subcrenata was the prismatic layer, and the frictional coefficient was the smallest. After 400s, the prismatic layer was exhausted. At that time, the nacreous layer resisted abrasion and the frictional coefficient increased. 
Before 200s, the friction surface was not completely contacted owing to the existence of radial ribs, so the wear rate was the smallest. From 600s, the wear rate reached the maximum degree due to lower hardness and higher toughness of the nacreous layer.

Table 1 Orthogonal test table

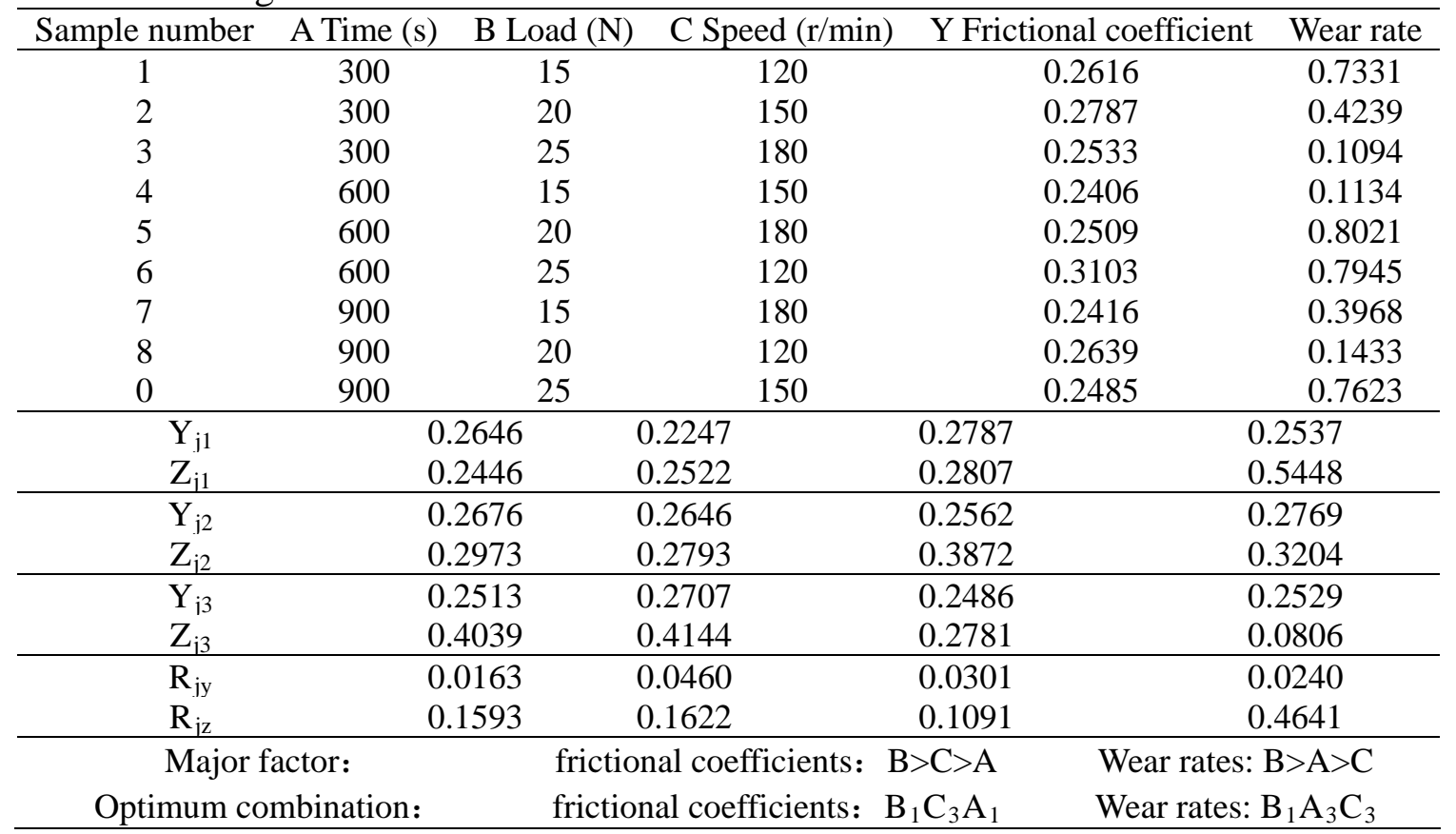

\section{Conclusions}

This paper has investigated microstructure and friction-wear behaviour of Scapharca subcrenata. According to the analyses, this study comes to the following conclusions:

1) When the nacreous layer contacted and rubbed with sandpaper, the frictional coefficient firstly became big and then declined with an increase in loads. The frictional coefficient dropped rapidly as sliding velocity increased. The higher the sliding speed was, the greater the normal load was and the smaller the frictional coefficient was.

2) The wear rate decreased with an increase in sliding speed. The wear rate was linearly related to the normal load. During the friction process of Scapharca subcrenata and sandpaper, the frictional coefficient was basically stable at around 0.26 . The cuticle obtained that highest wear resistance, the wear performances of the nacreous layer and the prismatic layer friction were relatively lower.

\section{Acknowledgement}

The authors would like to acknowledge the Project Doctor Scientific Research Startup Fund (No.2012.301) and the Project Seed Fund (No.2015.247) of Jilin Agricultural Science and Technology College, the Scientific Research Program Project of Jilin Provincial Education Department (JiJiaoKeHeZi.2015.No.378) , the Project Youth Scientific Research Fund (No.20150520107JH) of Jilin Province Science and Technology Development Program for financial support.

\section{References}

[1] Xian Jia, Xiaomei Ling, Donghai Tang. Microstructures and friction-wear characteristics of bivalve shells [J]. Tribology International, 2006 39(7)657-662.

[2] Limei Tian, Ximei Tian, Yinci Wang. Anti-wear properties of the molluscan shell Scapharca subcrenata: Influence of surface morphology, structure and organic material on the elementary wear 
process [J]. Materials Science and Engineering: C, 2014 (42)7-14.

[3] R. Arvind Singh, L. Siyuan, N. Satyanarayana. Bio-inspired polymeric patterns with enhanced wear durability for microsystem applications [J]. Materials Science and Engineering: C, 2011 31(7)1577-1583.

[4] Jin Tong, Mohammad Almagzoub Mohammad, Jinbo Zhang. DEM Numerical Simulation of Abrasive Wear Characteristics of a Bioinspired Ridged Surface [J]. Journal of Bionic Engineering, $20107(2) 175-181$.

[5] Philippe Stempflé, Marcel Brendlé. Tribological behaviour of nacre-Influence of the environment on the elementary wear processes [J]. Tribology International, 2006 39(12)1485-1496. 